\title{
Nonequilibrium Structural Condition in the Medical TiNi-Based Alloy Surface Layer Treated by Electron Beam
}

\author{
Aleksei A. Neiman ${ }^{1, \text { a) }}$, Ludmila L. Meisner ${ }^{1,2, b)}$, Aleksandr I. Lotkov ${ }^{1}$, \\ Nikolai N. Koval ${ }^{2,3}$, Viktor O. Semin ${ }^{1,2}$, and Anton D. Teresov ${ }^{2,3}$ \\ ${ }^{1}$ Institute of Strength Physics and Materials Science SB RAS, Tomsk, 634055, Russia \\ ${ }^{2}$ National Research Tomsk State University, Tomsk, 634050, Russia \\ ${ }^{3}$ Institute of High Current Electronics SB RAS, Tomsk, 634055, Russia

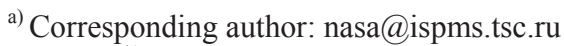 \\ b) meisner21@yahoo.com
}

\begin{abstract}
The research is devoted to study the structural condition and their evolution from the surface to the depth of TiNi specimens treated by low-energy high-current electron beams with surface melting at a beam energy density $E=$ $10 \mathrm{~J} / \mathrm{cm}^{2}$, number of pulses $N=10$, and pulse duration $\tau=50 \mu \mathrm{s}$. Determined thickness of the remelted layer, found that it has a layered structure in which each layer differs in phase composition and structural phase state. Refinement B2 phase lattice parameters in local areas showed the presence of strong inhomogeneous lattice strain.
\end{abstract}

Keywords: TiNi alloy, low-energy high-current pulsed electron beams, atomic crystal structure

\section{INTRODUCTION}

The way in which high-energy charged particle beams interact with materials and the applicability of this interaction in industry ensures much research interest [1-4]. The action of a high-energy pulse on the metal or alloy surface represents a single superfast thermal cycle of heating (melting) and cooling (quenching) with dynamic loading resulting in severe deformation [5]. All these processes in a single cycle change the chemical and phase compositions, giving rise to nonequilibrium microstructures, both in directly affected thin near-surface regions and in deeper materials layers [1-4]. The thus changed composition and surface layer structure provides improvement of physical, chemical, and mechanical properties of materials to a level often unattainable by other more widely used methods of surface treatment [6]. For example, low-energy high-current pulsed electron beam treatment of titaniumnickel (TiNi) alloy increases its corrosion resistance, biocompatibility, hardness, and fatigue life [7].

Research results on low-energy high-current pulsed electron beam treatment of different materials, mainly steels, [1-6, 8-9] show that the modified near-surface region can be divided into three successive layers differing in thickness: (1) a melted and rapidly quenched outer layer $\sim 1 \mu \mathrm{m}$ thick; (2) a high-temperature layer $\sim 10 \mu \mathrm{m}$ thick in which diffusion and diffusionless phase transformations, deformation, and recrystallization may occur; and (3) a layer of elastic wave action $\sim 100 \mu \mathrm{m}$ thick. Although it is generally recognized that the surface properties of material after low-energy high-current pulsed electron beam treatment are defined by the resulting state of its nearsurface microstructure and structural states formed in individual layers, there are still few studies that provide detailed structural analysis of these layers at different depths. Research data on microstructure evolution in surface melting and high-temperature heating zones of TiNi alloy irradiated by low-energy high-current pulsed electron beams can be found elsewhere [4, 10]. Analysis of the available data shows that the evolution of structural states in TiNi surface layers and their change depending on the parameters of pulsed electron beams are poorly studied and are thus not quite clear. In the work presented, we performed cross-sectional TEM analysis and X-ray diffraction (XRD) analysis of TiNi irradiated by low-energy high-current pulsed electron beams.

International Conference on Physical Mesomechanics of Multilevel Systems 2014

AIP Conf. Proc. 1623, 435-438 (2014); doi: 10.1063/1.4898975

(C) 2014 AIP Publishing LLC 978-0-7354-1260-6/\$30.00 
Thus, the objective of the work was to experimentally study the mechanisms by which nonequilibrium structural states are formed in near-surface layers of TiNi-based alloy under pulsed electron beam irradiation with surface melting used for surface treatment of medical implants.

\section{MATERIAL AND RESEARCH TECHNIQUES}

The test alloy of composition $\mathrm{Ti}_{49.5} \mathrm{Ni}_{50.5}$ was melted from iodide titanium and $\mathrm{N} 0$-grade nickel in an electric arc furnace with six-fold remelting of the ingot. The ingot was homogenized at $T=1073 \mathrm{~K}$ for $3 \mathrm{~h}$ and cooled in the furnace. The start and finish temperatures of direct and reverse martensitic transformations were $M_{\mathrm{s}}=290 \mathrm{~K}, M_{\mathrm{f}}=$ $270 \mathrm{~K}, A_{\mathrm{s}}=303 \mathrm{~K}$ and $A_{\mathrm{f}}=330 \mathrm{~K}$. The extruded ingot was cut by electroerosion cutting perpendicular to the bar axis to obtain TiNi specimens in the form of disks of thickness $1 \mathrm{~mm}$ and diameter $13 \mathrm{~mm}$ which were then annealed in vacuum at $T=1073 \mathrm{~K}$ and $P=10^{-3} \mathrm{~Pa}$ for $1 \mathrm{~h}$ and cooled in the furnace.

Before electron beam irradiation, the surface of all specimens was subjected to the following treatment: (1) chemical etching in acid solution $\left(\mathrm{HNO}_{3} / \mathrm{HF}=3 / 1\right)$; (2) mechanical grinding and high luster polishing and (3) electropolishing in acid solution $\left(\mathrm{CH}_{3} \mathrm{COOH} / \mathrm{HClO}_{4}=3 / 1\right)$. The specimens subjected to the foregoing preliminary surface treatment are hereinafter referred to as nonirradiated or initial specimens.

The specimens were irradiated by low-energy high-current electron beams on a SOLO setup (Institute of High Current Electronics SB RAS, Tomsk) at an energy density $E=10 \mathrm{~J} / \mathrm{cm}^{2}$, number of pulses $N=10$, pulse duration $\tau=$ $50 \mu \mathrm{s}$, and pulse repetition frequency $v=0.3 \mathrm{~Hz}$. The working gas (argon) pressure in the chamber was $P_{\mathrm{Ar}}=$ $3.5 \times 10^{-2} \mathrm{~Pa}$.

XRD analysis (XRD) analysis of structural phase states in TiNi surface layers before and after irradiation was performed on a DRON-7 diffractometer (Burevestnik, Russia) in Co-K $\mathrm{K}_{\alpha}$ radiation.

The procedure of preparing the electron beam-treated specimens in cross-section dimension for TEM analysis for ion etching, an EM 09100IS ion slicer (JEOL, Japan) was used. The microstructure of TiNi surface layers before and after electron beam treatment was examined by TEM with a JEM 2100 transmission electron microscope (JEOL, Japan). For decode electron diffraction patterns of the TiNi specimens, a special program in the Mathcad environment was developed. In the interpretation, data on the structure of all phases characteristic of the $\mathrm{Ti}-\mathrm{Ni}$ system were used. The developed program allows one to determine the indices of crystallographic planes of individual phases and to calculate their lattice parameters.

\section{RESEARCH RESULTS AND DISCUSSION}

According the data of XRD analysis, the volume fraction of the $\mathrm{Ti}_{2} \mathrm{Ni}$ phase $(\nabla)$ with fcc structure in the initial specimen (Fig. 1(a)) is $\leq 5$ vol. \%; the other volume ( $\geq 95$ vol.\%) belongs to the high-temperature B2 phase $(\diamond)$ with $\mathrm{CsCl}$-ordered structure, and composition close to equiatomic.

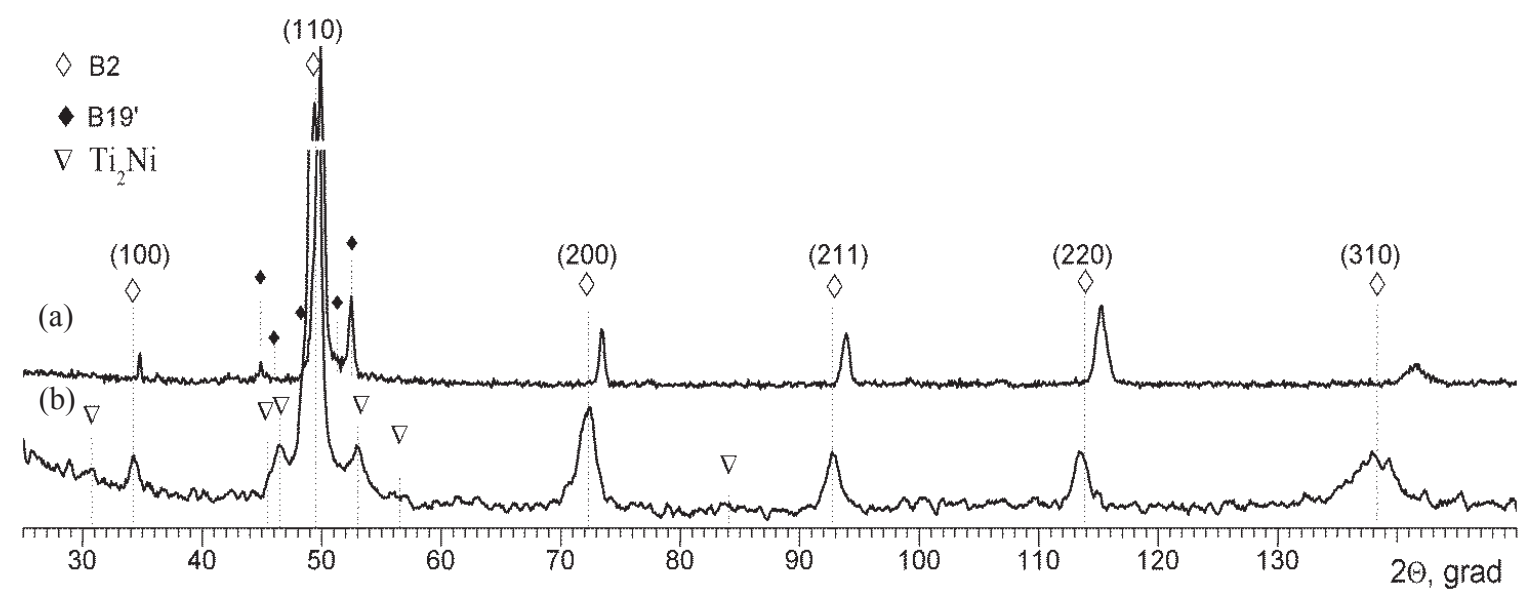

FIGURE 1. Diffraction patterns for TiNi before (a) and after electron beam treatment (b). Symmetric geometry, Co-K radiation 

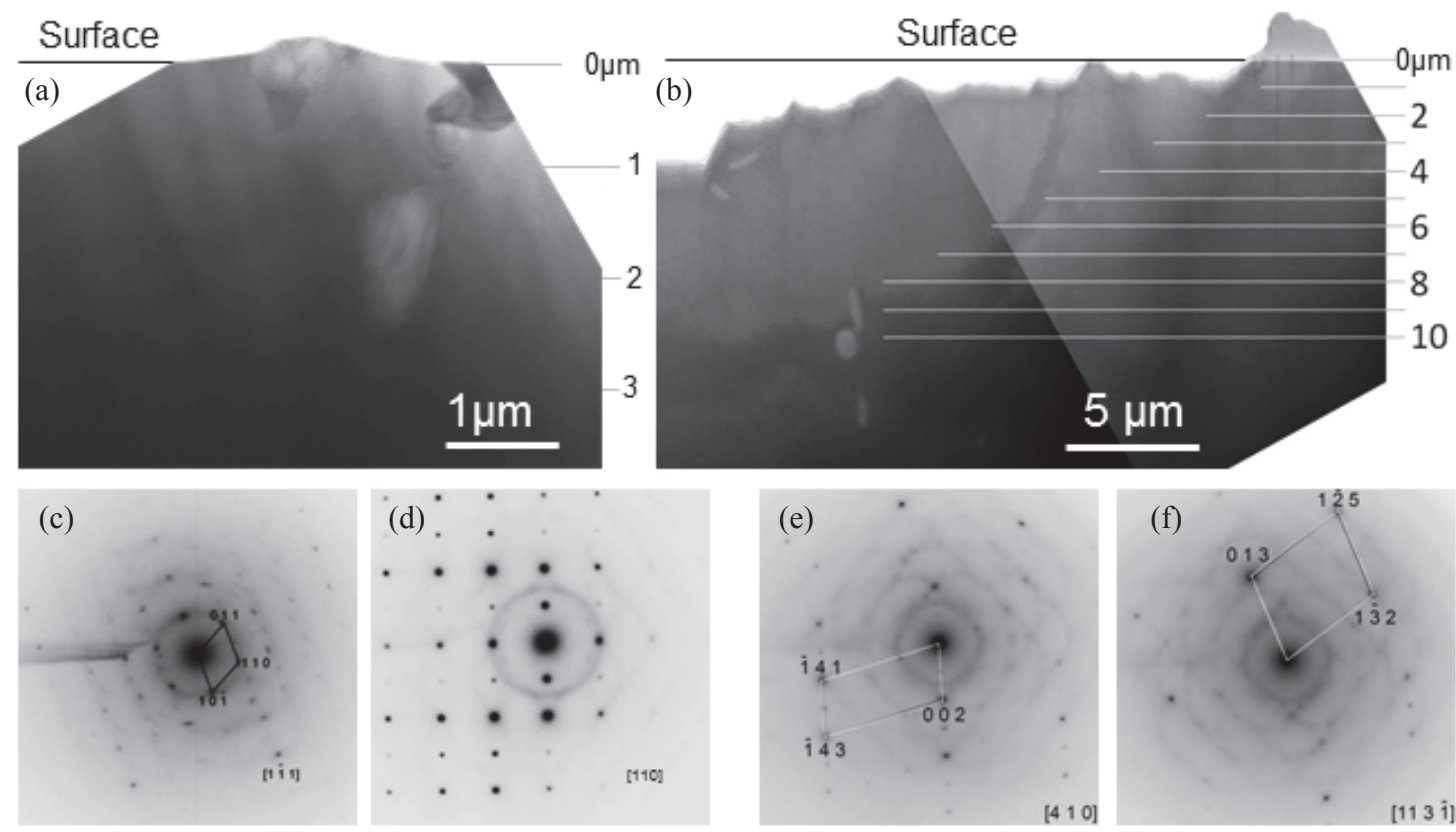

FIGURE 2. Bright-field image (a, b) and microdiffraction patterns (c-f) from the TiNi surface before $(a, c, d)$ and after $(b, e, f)$ electron beam treatment

The lattice parameter of the initial B2 phase, according to the XRD data, is $a_{\mathrm{B} 2}^{\mathrm{TiNi}}=3.0139 \pm 0.0008 \AA$ that corresponds to $\mathrm{Ti}_{49.5} \mathrm{Ni}_{50.5}$. In the $\mathrm{B} 2$ phase of this composition, a $\mathrm{B} 2 \rightarrow \mathrm{B} 19^{\prime}$ martensite transformation is realized. The start and finish temperatures of direct and reverse martensite transformations in the alloy are in the vicinity of room temperature. On X-ray diffraction patterns at $T=293 \mathrm{~K}$, no B19' martensitic phase ( $\downarrow)$ with monoclinic structure was observed in the TiNi specimen before irradiation.

After electron beam irradiation, the X-ray diffraction pattern (Fig. 1(b)) reveals peaks of the B2 phase $(\diamond)$ with $\leq 95$ vol.\% $a_{\mathrm{B} 2}^{\mathrm{TiN}_{\mathrm{ir}}}=3.0283 \pm 0.0013 \AA$ and $\mathrm{B} 19^{\prime}$ phase $(\diamond)$ with $\geq 5$ vol.\%. Peaks of the $\mathrm{Ti}_{2} \mathrm{Ni}$ phase $(\nabla)$ on the $\mathrm{X}-$ ray pattern are almost absent (Fig. 1(b)).

According to the data of transmission electron microscopy, particles of the $\mathrm{Ti}_{2} \mathrm{Ni}$ phase in the initial specimen (Fig. 2(a)) are found both in the material volume and on the surface. After electron beam treatment (Fig. 2(b)), Ti ${ }_{2} \mathrm{Ni}$ particles are found only at a depth of $8-10 \mu \mathrm{m}$ from the surface and are almost absent in the modified near-surface layer. The absence of $\mathrm{Ti}_{2} \mathrm{Ni}$ particles in the modified layer evidences that the chosen electron beam treatment mode did induce melting of the TiNi surface layer and that the $\mathrm{Ti}_{2} \mathrm{Ni}$ particles were melted earlier than the main $\mathrm{B} 2$ phase because of the lower melting temperature of $\mathrm{Ti}_{2} \mathrm{Ni}$ compared to that of the main $\mathrm{B} 2$ phase.

On electron beam treatment with $N=10$ pulses, the local heterogeneities of the chemical composition in the regions of dissolved $\mathrm{Ti}_{2} \mathrm{Ni}$ particles are likely to be quasi-uniformly mixed with the melted TiNi matrix and elemental composition in this region corresponds to $\mathrm{Ti}_{51.7} \mathrm{Ni}_{48.3}$.

The X-ray methods fail to give details of the structural state in the layer modified by electron beam melting and subsequent quenching. Therefore, the lateral foil sections (see Materials and Research Techniques) were subjected to TEM analysis in which the B2 and B19' lattice parameters obtained from XRD data were used. It is found that the electron diffraction patterns of the B2 phase at any depth of the nonirradiated TiNi specimen can unambiguously be interpreted using the X-ray value of the $\mathrm{B} 2$ lattice parameter $a_{\mathrm{B} 2}^{\mathrm{TiNi}} \cong 3.014 \AA$.

On the contrary, the diffraction patterns of the modified TiNi layer (Fig. 2(e, f)) are impossible to interpret using the X-ray value of the $\mathrm{B} 2$ lattice parameter both in the initial state $a_{\mathrm{B} 2}^{\mathrm{TiNi}} \cong 3.014 \AA$ and in the state after electron beam treatment $a_{\mathrm{B} 2}^{\mathrm{TiNi}_{\mathrm{ir}}}=3.0283 \AA$ because the positions of its actual peaks (solid circles) are noncoincident with those calculated from X-ray data. 
TABLE 1. Atomic crystal structure parameters in local TiNi near-surface regions after electron beam treatment. Microdiffraction interpretation

\begin{tabular}{lcccccc}
\hline $\begin{array}{l}\text { Surface to Diffraction Region } \\
\text { Distance } \boldsymbol{h} \boldsymbol{\mu} \mathbf{m}\end{array}$ & 0.5 & 1 & 2 & 7 & $10-20$ & $>20$ \\
\hline B2 Phase Elementary Cell & $a=3.003 \AA$ & $a=3.033 \AA$ & $a=3.013 \AA$ & $a=3.033 \AA$ & $a=3.013 \AA$ & $a=3.013 \AA$ \\
Parameters in Electron Beam- & $b=3.003 \AA$ & $b=3.033 \AA$ & $b=3.013 \AA$ & $b=3.033 \AA$ & $b=3.013 \AA$ & $b=3.013 \AA$ \\
Treated Near-Surface Layers & $c=3.063 \AA$ & $c=3.063 \AA$ & $c=3.033 \AA$ & $c=3.063 \AA$ & $c=3.023 \AA$ & $c=3.013 \AA$ \\
& $\alpha=89.5^{\circ}$ & $\alpha=89.3^{\circ}$ & $\alpha=89.8^{\circ}$ & $\alpha=90^{\circ}$ & $\alpha=90^{\circ}$ & $\alpha=90^{\circ}$ \\
& $\beta=90^{\circ}$ & $\beta=90^{\circ}$ & $\beta=90^{\circ}$ & $\beta=90^{\circ}$ & $\beta=90^{\circ}$ & $\beta=90^{\circ}$ \\
& $\gamma=90^{\circ}$ & $\gamma=90^{\circ}$ & $\gamma=90^{\circ}$ & $\gamma=90^{\circ}$ & $\gamma=90^{\circ}$ & $\gamma=90^{\circ}$ \\
\hline Atomic Volume $\boldsymbol{V}_{\text {TEM }}, \AA^{\mathbf{3}}$ & 13.811 & 14.087 & 13.767 & 14.088 & 13.767 & 13.676 \\
\hline Atomic Volume $\boldsymbol{V}_{\text {XRD }}, \AA^{\mathbf{3}}$ & 13.882 & 13.882 & 13.882 & 13.882 & 13.722 & 13.676 \\
\hline
\end{tabular}

The structural parameters corresponding to the experimentally observed peaks on electron diffraction patterns were calculated using general crystallographic form with elementary cell parameters $a \neq b \neq c$ and angles between elementary translations $\neq \beta \neq \gamma$ the values of which were varied in the Mathcad program. The calculated data are presented in Table 1.

Analysis of the data in Table 1 suggests the following: (1) in the modified layer at different depths from the surface, the electron diffraction pattern preserves typical diffraction features of cubic structure (no splitting, no doublets or new peaks); (2) the structure of the main phase in this layer is however impossible to describe with only one value of the lattice parameter and one of the quadrant angles deviates from $90^{\circ}$; (3) there is no strict regularity in variation of the structural parameters depending, for example, on the location depth of the region (with respect to the irradiated surface) from which diffraction is obtained; and (4) the above peculiarities are well pronounced in the outer layer of thickness $\sim 10 \mu \mathrm{m}$ subjected to melting and are damped with depth from the irradiated surface such that the diffraction at a depth larger than $20 \mu \mathrm{m}$ corresponds to the B2 phase structure with its initial lattice parameter $a_{\mathrm{B} 2}^{\mathrm{TiNi}} \cong 3.014 \AA$.

\section{REFERENCES}

1. A. D. Pogrebnjak, V. T. Shablya, N. V. Sviridenko, et al., Surf. Coat. Technol. 111(1), 46 (1999).

2. M. Sorescu, J. Alloy Compd. 280(1-2), 251 (1998).

3. A. B. Markov and V. P. Rotshtein, Nucl. Instrum. Meth. Phys. Res. B: Beam Interact. Mater. Atoms 132(1), 79 (1997).

4. K. M. Zhang, J. X. Zou, T. Grosdidier, et al., J. Vac. Sci. Technol. A. Vac. Surf. Films 25(1), 28 (2007).

5. K. M. Zhang, J. X. Zou, B. Bolle, and T. Grosdidier, Vacuum 87, 60 (2013).

6. A. D. Pogrebnjak, V. S. Ladysev, N. A. Pogrebnjak, et al., Vacuum 58(1), 45 (2000).

7. L. L. Meisner, I. V. Nikonova, A. I. Lotkov, et al., Perspekt. Mater., 3, 15 (2011).

8. Q. Guan, Q. Zhang, and C. Dong, ISIJ Int. 48(2), 235 (2008).

9. K. Zhang, J. Zou, T. Grosdidier, and C. Dong, J. Vac. Sci. Technol. A. Vac. Surf. Films 28(6), 1349 (2010).

10. J. X. Zou, T. Grosdidier, K. M. Zhang, C. Dong, and S. Weber, Eur. Phys. J. Appl. Phys. 43(3), 327 (2008). 